\title{
Análisis Comparativo de los Programas de Pregrado en Tecnología en Salud Ocupacional y afines en Colombia
}

\author{
Comparative Analysis of the Undergraduate Programs in Technology in Occupational and \\ Related Health in Colombia \\ ${ }^{a}$ Johon Gutiérrez Jaraba ${ }^{87}$, Fabio Pérez Márquez ${ }^{b}$, Rocio Vergara de la Ossa ${ }^{c}$ \\ a johon.gutierrez@unitecnar.edu.co “Director de Investigación e Innovación, Fundación Universitaria Antonio de Arévalo - UNITECNAR, \\ Cartagena, Colombia. \\ b fabio.perez@unitecnar.edu.co "Coordinador Editorial, Fundación Universitaria Antonio de Arévalo - UNITECNAR, Cartagena, Colombia.

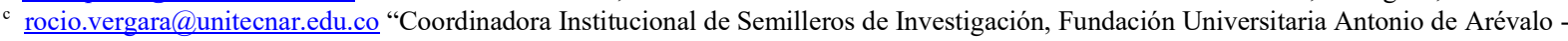 \\ UNITECNAR, Cartagena, Colombia.
}

Forma de Citar: J. Gutiérrez-Jaraba, F. Pérez-Márquez et al, “Análisis Comparativo de los Programas de Pregrado en Tecnología en Salud Ocupacional y afines en Colombia", Rev. Saberes, Vol. 13, No. 02, pp. 120 - 127, 2020.

Recibido: 24/03/2020 Evaluación: 28/05/2020 Aceptado: 30/06/2020 DOI: https://doi.org/1025213/1794-4384/1302.017

\section{Resumen}

El presente trabajo, tuvo por objetivo realizar un análisis comparativo de las carreras de Tecnología en Salud Ocupacional en Cartagena, la Costa Atlántica y Colombia. El trabajo fue apoyado por la Dirección de Investigación e Innovación de la Fundación Universitaria Antonio de Arévalo UNITECNAR y su grupo de investigación Desarrollo Sostenible Urbano. El alcance del estudio comprendió la revisión de la oferta educativa desde el nivel mencionado con base en la información reportada en la plataforma del Sistema Nacional de Información de la Educación Superior - SNIES del Ministerio de Educación Nacional en Colombia - MEN al año 2018. En concreto, el trabajo buscó mostrar el estado actual de la formación en el campo de la profesión para lo cual comparó múltiples variables para los programas existentes por regiones, visualizando las tendencias generales.

Para conseguir el objetivo, se utilizaron los métodos y técnicas estadísticas de análisis descriptivo, modelos lineales generalizados y análisis de componentes principales. Entre los resultados, se muestra que los programas así denominados, están dentro de los parámetros académicos nacionales e internacionales y que su denominación es coherente con la naturaleza del campo de conocimiento al cual pertenecen con su respectivo nivel académico, además del grupo de características que los identifican y constituyen particularidades. También se muestra la necesidad y pertinencia de programas de esa denominación en la ciudad de Cartagena y resalta la existencia de programas similares en mayor cuantía en ciertas regiones del país, principalmente en la zona andina, lo cual justifica la necesidad de formación en la disciplina en el nivel del programa analizado.

\section{Palabras Clave}

Currículo, competencia, Ciclo propedéutico, educación.

\begin{abstract}
The objective of this work was to carry out a comparative analysis of the Careers of Technology in Occupational Health in Cartagena, the Atlantic Coast and Colombia. The work was supported by the Scientific and Technological Research of the Antonio de Arévalo University Foundation UNITECNAR and its Urban Sustainable Development research group. The scope of the
\end{abstract}

${ }^{87}$ Autor para correspondencia: correo electrónico johongutierrez@hotmail.com 
study included the revision of the educational offer at that level based on the information reported on the platform of the National Information System of Higher Education - SNIES of the Ministry of National Education in Colombia - MEN to the year 2016. Specifically, The work sought to show the current state of training in the field of the profession for which it compared multiple variables for existing programs by region, visualizing general trends.

To achieve the objective, the statistical methods and techniques of descriptive analysis, generalized linear models and principal component analysis were used. Among the results, it is shown that the programs so named are within the national and international academic parameters and that their name is consistent with the nature of the field of knowledge to which they belong with their respective academic level, in addition to the group of characteristics that the they identify and constitute particularities. It also shows the need and relevance of the program in the city of Cartagena and highlights the existence of similar programs to a greater extent in certain regions of the country, mainly in the Andean area, which justifies the need for training in the discipline at the level of the analyzed program.

\section{Keyword}

Curriculum, competition, propedeutic cycle, education.

\section{Introducción}

En el contexto mundial se están presentando grandes transformaciones producto de la revolución científica y el uso intensivo de las nuevas tecnologías de la información y comunicación que afectan directamente a la sociedad colombiana y por ende, al sistema educativo. Estos grandes cambios plantean retos a la educación superior exigiendo ofrecer programas pertinentes y con calidad que permitan contribuir al desarrollo de la región y el país. No en vano se reconoce que la educación mantiene un importante rol estratégico y prioritario en el desarrollo humano, social y económico, por lo que exige especial atención. [1].
Así las cosas, con el fin de responder al derrotero anteriormente planteado, los gobiernos y sectores educativos y empresariales, buscan trabajar mancomunadamente con el fin de lograr construir de forma conjunta políticas $y$ estrategias encaminadas al fortalecimiento de la formación de profesionales en las nuevas tendencias disciplinares que respondan con pertinencia a las necesidades sectoriales. [2].

Al respecto de la eficacia, uno de los problemas detectados ha sido la tendencia progresiva de continuar formando profesionales universitarios en carreras tradicionales, cuando los sectores productivos insistentemente plantean la necesidad de formar individuos con competencias y destrezas en hacer y saber hacer. La actual tasa de desempleo en el país y el desempeño laboral en actividades ajenas a los campos de formación del nivel universitario, son una muestra evidente de la saturación del mercado en el ámbito de algunas profesiones por lo que se hace imperativo identificar las reales necesidades de formación que requieren el país y sus regiones con el fin de alcanzar los objetivos de crecimiento proyectados. [3].

No es desconocido que la educación superior en Colombia enfrenta retos importantes dentro de los que sobresalen el aumento de niveles de cobertura y el mejoramiento de la calidad de las instituciones que ofrecen los servicios en ese nivel de enseñanza. Si bien, durante las dos últimas décadas el número de estudiantes matriculados ha crecido de manera importante, especialmente en la formación técnica y tecnológica, en un contexto internacional las tasas de cobertura continúan siendo bajas y no superan el $50 \%$. Por otro lado, la calidad del sistema de educación superior es heterogénea, ya que coexisten instituciones bien organizadas y reconocidas por su excelencia, con instituciones caracterizadas por bajos niveles de calidad. [4].

\section{Marco Legal}

Al respecto del marco legal de la educación superior para carreras técnicas y tecnológicas en Colombia, debe decirse que la Constitución Política de 1991 establece que la educación es un 
derecho fundamental, la cual tiene carácter obligatorio en los nueve primeros años escolares siendo seguida por la educación media la cual es antesala de la educación superior, conocida como el nivel superior de la estructura educativa nacional. Por su parte, es el Ministerio de Educación Nacional (MEN) la entidad encargada de articular el sistema de educación a partir de políticas que comprenden desde los niveles básicos de preescolar hasta llegar a la educación profesional de alto nivel, la cual es competencia del Viceministerio de Educación Superior que mediante las funciones de fomento, inspección y vigilancia, orienta la educación superior con el fin de garantizar el ingreso y la permanencia de los estudiantes en este nivel. Cabe decir que la educación superior colombiana abarca la técnica, la ciencia y la tecnología, las humanidades, el arte y la filosofía en dos niveles, pregrado y postgrado y a su vez, el pregrado ofrece tres niveles de formación, técnica profesional, tecnológica y profesional universitaria, y el postgrado, especialización, maestría y doctorado. [5].

Así mismo y refiriéndose al mismo nivel de carreras técnicas y tecnológicas, se informa que el marco legal está constituido básicamente por la Ley 30 de 1992 que organiza el servicio público de la educación superior estableciendo que son aceptadas como establecimientos de la educación superior las Instituciones Técnicas Profesionales, las Instituciones Universitarias o Escuelas Tecnológicas y las Universidades. Esa misma ley concibe la educación "como un proceso permanente que posibilita el desarrollo de las potencialidades del ser humano de una manera integral y tiene por objeto el desarrollo pleno de los alumnos y su formación académica o profesional" y que debe "Profundizar en la formación integral, trabajar por la creación, el desarrollo y la transmisión del conocimiento; promover la utilización del conocimiento para solucionar las necesidades del país y prestar a la comunidad un servicio con calidad. Además, ser factor de desarrollo científico, cultural, económico, político y ético".

Por su parte la Ley 749 de 2002 que también regula los niveles anteriormente referenciados y "por la cual se organiza el servicio público de la educación superior en las modalidades de formación técnica profesional y tecnológica", contiene los lineamientos por los cuales se rigen tanto los programas en las modalidades técnicas profesionales y tecnológicas y las respectivas instituciones de educación superior que se caracterizan por su vocación e identidad manifiesta en los campos de los conocimientos y el trabajo en actividades de carácter técnico, debidamente fundamentadas en la naturaleza de un saber, cuya formación debe garantizar la interacción de lo intelectual con lo instrumental, lo operacional y el saber técnico.

En la misma Ley 749, se encuentran definidos claramente los ciclos de formación, expresando claramente que las instituciones técnicas profesionales y tecnológicas de educación superior organizarán su actividad formativa de pregrado en niveles educativos y ciclos propedéuticos así:

a) El Primer Nivel, estará orientado a generar competencias y desarrollo intelectual como el de aptitudes, habilidades y destrezas al impartir conocimientos técnicos necesarios para el desempeño laboral en una actividad, en áreas específicas de los sectores productivos $\mathrm{y}$ de servicios, que conducirá al título de Técnico Profesional.

La formación técnica profesional comprende tareas relacionadas con actividades técnicas que pueden realizarse autónomamente, habilitando para comportar responsabilidades de programación y coordinación.

b) El Segundo Nivel, ofrecerá una formación básica común, que se fundamente y apropie de los conocimientos científicos y la comprensión teórica para la formación de un pensamiento innovador e inteligente, con capacidad de diseñar, construir, ejecutar, controlar, transformar y operar los medios y procesos que han de favorecer la acción del hombre en la solución de problemas que demandan los sectores productivos y de servicios del país. La formación tecnológica comprende el desarrollo de responsabilidades de concepción, dirección y gestión de conformidad con la especificidad del programa, y conducirá al título de Tecnólogo en el área respectiva.

c) El Tercer Nivel, complementará el segundo ciclo, en la respectiva área del conocimiento, de forma coherente, con la fundamentación teórica y 
la propuesta metodológica de la profesión, y debe hacer explícitos los principios y propósitos que la orientan desde una perspectiva integral, considerando, entre otros aspectos, las características y competencias que se espera posea el futuro profesional. Este ciclo permite el ejercicio autónomo de actividades profesionales de alto nivel, e implica el dominio de conocimientos científicos y técnicos y conducirá al título de Profesional.

Finalmente se debe informar que el sistema tiene sus reguladores, evaluadores, asesores y prestadores de servicio público. Como reguladores pueden considerarse el Congreso de la República, el Ministerio de Educación Nacional y el Departamento Nacional de Planeación. Estas entidades cumplen funciones de evaluación, además de otros organismos como el Consejo Nacional de Educación Superior - CESU, Consejo Nacional de Acreditación - CNA, Consejo Nacional de Aseguramiento de la Calidad de la Educación Superior - CONACES y los pares académicos. Por su parte, los prestadores del servicio público son las entidades de educación superior que pueden ser públicas y privadas.

\section{Situación Actual de la Educación Técnica y Tecnológica en Colombia.}

La distribución de la matrícula en Colombia con respecto a la registrada en algunos países de Latinoamérica, presenta grandes diferencias. Según cifras de la UNESCO, en Chile y Uruguay la matrícula en programas técnicos y tecnológicos supera a la del nivel universitario. Si se compara esta proporción con países de Europa como Francia, Reino Unido y Países Bajos, la diferencia se amplía significativamente a favor de la formación técnica profesional y tecnológica.[6]

La respuesta sobre cuál distribución es la más favorable para el desarrollo económico y social de un país debe coincidir con los requerimientos del sector productivo y de la brecha que exista en la distribución del ingreso de sus habitantes. En Colombia se evidencia la necesidad de contar con un mayor número de técnicos profesionales $\mathrm{y}$ tecnólogos que se especialicen en los sectores que demanda el país para incrementar su productividad y mejorar su competitividad, y al mismo tiempo, que este mayor volumen de población incorporada al mercado laboral genere ingresos que contribuyan a disminuir la pobreza y a cerrar la brecha en la distribución del ingreso.

En ese orden de ideas, el Ministerio de Educación Nacional - MEN, ha venido planteando que para "Aceptar el desafio de contribuir a impulsar el fortalecimiento de la educación técnica y tecnológica y con ello la competitividad y la productividad del país, se requiere conocer el contexto en el que se formulan e implementan las políticas nacionales en el sector educativo”. [6]

El mismo MEN expresa que para la oferta de estos programas, el país contó en el año 2008 con 276 instituciones de educación superior que, de acuerdo con la Ley 30 de 1992, se clasificaron, según su carácter, en cuatro grupos: instituciones técnicas profesionales, institutos tecnológicos, instituciones universitarias y universidades. De estas 276 instituciones, 195 eran privadas $(71 \%)$ y 81 públicas $(29 \%)$; el $33 \%$ del total eran instituciones universitarias, el $26.8 \%$ universidades, el $21.7 \%$ institutos tecnológicos y el restante $18.5 \%$, instituciones técnicas profesionales. [7]

En el mismo año, la oferta de la educación superior en Colombia abarcó 55 núcleos básicos del conocimiento en los que se agruparon 5.581 programas de pregrado (706 técnicos profesionales, 1.407 tecnológicos y 3.468 universitarios). Sin embargo, la demanda se concentró en 20 núcleos y de éstos es evidente una preferencia de los estudiantes por carreras tradicionales como Derecho, Administración de Empresas, Contaduría Pública y Medicina.

Históricamente ha existido en el país una preferencia de los estudiantes por los programas universitarios. Un seguimiento a la matrícula en educación superior en los últimos tres años permitió observar una concentración de la matrícula en programas universitarios $(74.6 \%)$ frente a programas técnicos profesionales $\mathrm{y}$ tecnológicos $(20,7 \%)$. Para contrarrestar los efectos, el Plan Sectorial de Educación 2006-2010 propuso la meta de crear 320.000 cupos en educación superior, de los cuales 200.000 correspondieron a formación técnica y tecnológica, de tal manera que la participación de 
este nivel en el total de la matrícula de educación superior pasó de $25,7 \%$ en 2006 a $34 \%$ en 2010. [8].

\section{Metodología}

El alcance del estudio comprendió la revisión de la oferta educativa del nivel tecnológico en el área de gestión financiera en Cartagena, Costa Atlántica y Colombia con base en la información reportada en la plataforma del Sistema Nacional de Información de la Educación Superior - SNIES del Ministerio de Educación Nacional en Colombia - MEN al año 2016.

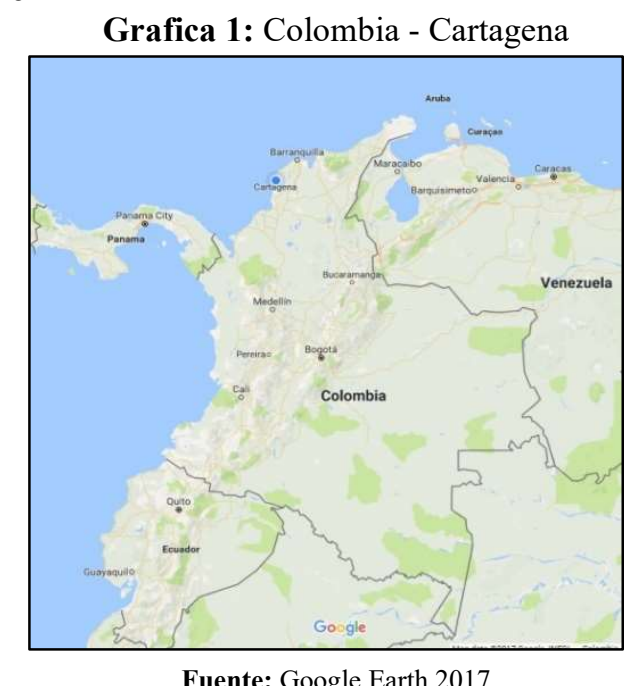

A continuación, se describen las etapas del procesamiento de datos que permitió realizar el análisis comparativo de la carrera de nivel tecnológico en estudio.

\section{- Procesamiento de la Información}

a) La información correspondiente a las carreras relacionadas con Tecnología en Salud Ocupacional, se recopiló del SNIES, sistema que recopila y organiza la información relevante sobre la educación superior que permite hacer planeación, monitoreo, evaluación, asesoría, inspección y vigilancia del sector. Este sistema como fuente de información, en relación con las instituciones y programas académicos aprobados por el Ministerio de Educación Nacional, consolida y suministra datos, estadísticas e indicadores considerados como relevantes, confiables y oportunos. De igual forma se contrastó aleatoriamente la existencia de las carreras en las páginas web de las instituciones que los reportan.

b) A continuación se procedió a realizar las gráficación individual con las respectivas valoraciones obtenidas al año 2018. Se ubicaron los programas activos e inactivos, distribución por sectores; metodologías, ciclos propedéuticos, cantidad de créditos, carácter de instituciones ofertantes, distribución por departamentos y costo entre otras variables.

c) Finalmente se organizó la información y se procedió con el análisis comparativo por cada gráfica generada por los modelos institucionales en el periodo anteriormente referenciado.

\section{Resultados}

- Análisis de carreras tecnológicas relacionados con el área Salud Ocupacional.

a) Distribución nacional del programa $(\text { TSO })^{88} y$ afines.

A nivel de distribución nacional, se encontró que de los 26 programas tecnológicos afines reportados en el SNIES, 22 se encuentran activos y 4 inactivos.

Grafica 2: Distribución Nal de programas. Col. 2018.

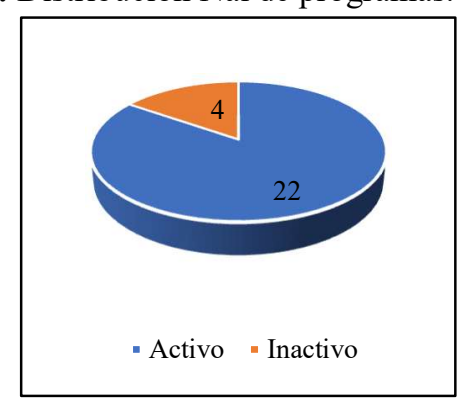

Fuente: SNIES, Colombia. 2018

b) Programa (TSO) frente a otras denominaciones del área.

\footnotetext{
${ }^{88}$ Tecnología en Salud Ocupacional
} 
Grafica 3: (TSO) vs Programas Afines. Col. 2018

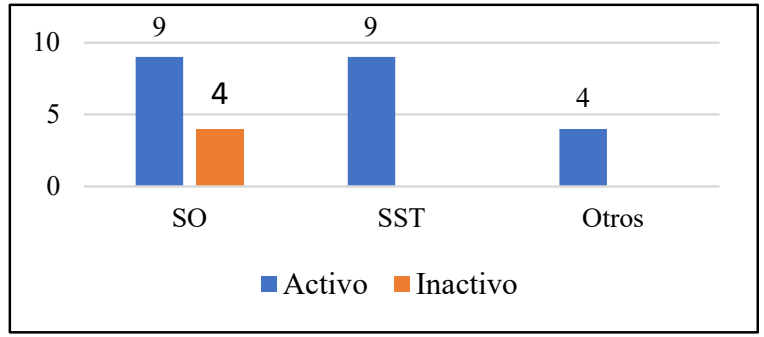

Fuente: SNIES, Colombia. 2018

Se encontró que del total de los 26 programas relacionados con el área de estudio a nivel nacional, solo 13 corresponden a denominaciones específicas del programa de Tecnología en Salud Ocupacional de los cuales nueve (9) se encuentran activos. Adicionalmente se pudo observar que los 13 restantes corresponden a denominaciones similares y afines, encontrándose activos 13 programas.

\section{c) Distribución Nacional (TSO) activos - regiones}

Grafica 4: (TSO) Distribución regional. Colombia. 2018

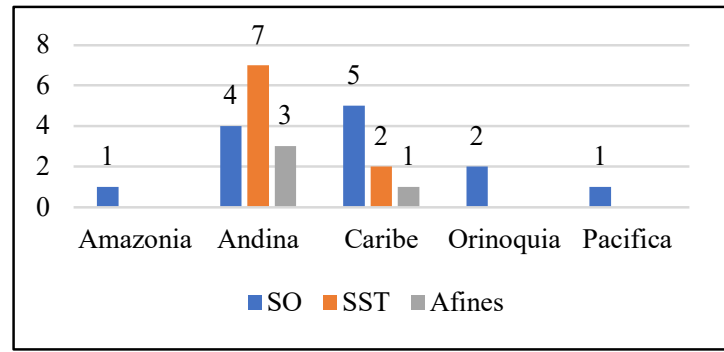

Fuente: SNIES, Colombia. 2018

En cuanto a la distribución nacional del programa de (TSO) en las cinco (5) regiones del país, se encontró uno (1) en la región de la Amazonia, cuatro (4) en la Andina, cinco (5) en la Caribe, dos (2) en la Orinoquia y uno (1) en la Pacífica.

\section{d) Modalidad Empleada por el Programa Activo} (TSO) a Nivel Nacional.

Grafica 5: (TSO) Modalidad. Colombia. 2018

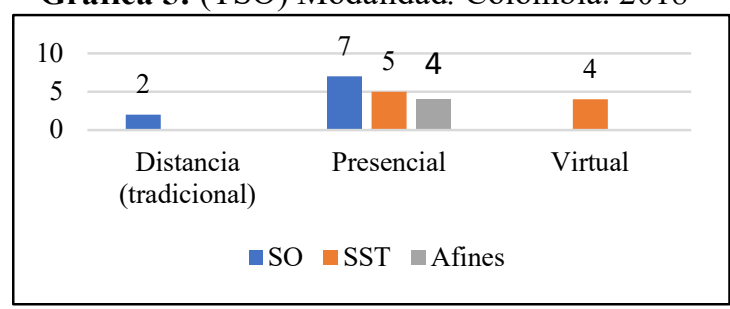

Fuente: SNIES, Colombia. 2018
De acuerdo a la información reportada en SNIES, se pudo observar que de los 22 programas de (TSO) activos a nivel nacional, siete (7) corresponden a la modalidad presencial; dos (2) a distancia tradicional.

e) Modalidad del programa activos (SST) por regiones.

Grafica 6: (SST) Modalidad por regiones. Col. 2018

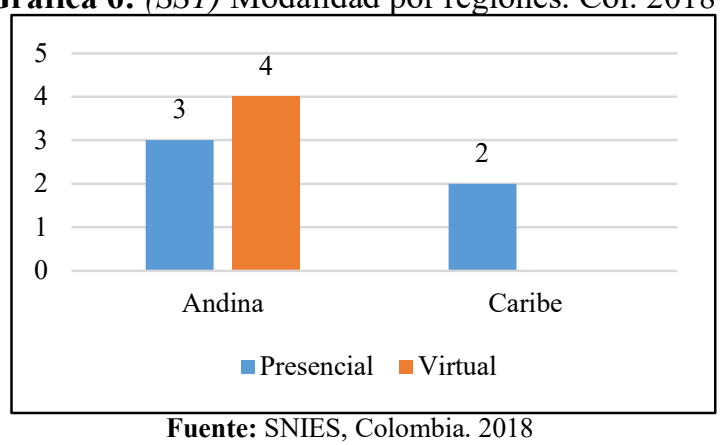

En cuanto a la información relacionada con la modalidad empleada por los programas activos (SST) a nivel de regiones, se encontró que solo es ofrecida en la región andina, con tres (3) en modalidad presencial y cuatro (4) virtual. Y en la región caribe con dos (2) Presenciales.

\section{f) Sectorización Programas Activos (SST) en Colombia.}

Grafica 7: (SST) por sector oferente. Col. 2018

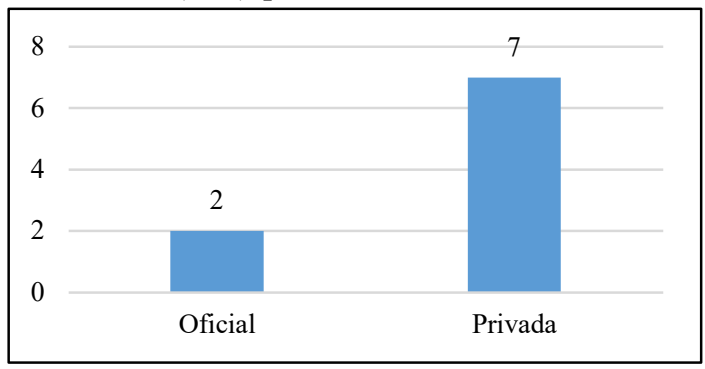

Fuente: SNIES, Colombia. 2018

La sectorización relacionada con un programa académico de cualquier nivel, está relacionada con el tipo de institución educativa que lo ofrece. Así las cosas, se encontró que el programa (SST) a nivel nacional en su gran mayoría es ofertado por instituciones privadas. En concreto se observó que de los nueve (9) programas (TSO) activos, siete (7) son de carácter privado y solamente dos corresponden al sector (2) oficial. 
g) Costos Matricula por Semestre Programas (SST) Activos en Colombia.

El costo de la matrícula es un factor que incide en la cobertura de las carreras en Colombia. Sin embargo, a la hora de querer estudiar una profesión no basta con tener la capacidad económica, sino que ello queda sujeto a la existencia de la carrera deseada.

Respecto a esta variable, se encontró que una (1) de las instituciones oficiales reportan el costo del semestre por menos de $\$ 0.6$ Millones y las otras dos (2) no reportan este valor. En cuanto a las privadas, cuatro (4) no reportan el valor y solo dos (2) instituciones mostraron costos que oscilan entre $\$ 1.4$ y $\$ 1.8$ millones.

h) Programas (SST) activos por ciclos propedéuticos en Colombia

Grafica 8: (SST) Ciclos propedéuticos. Col. 2018

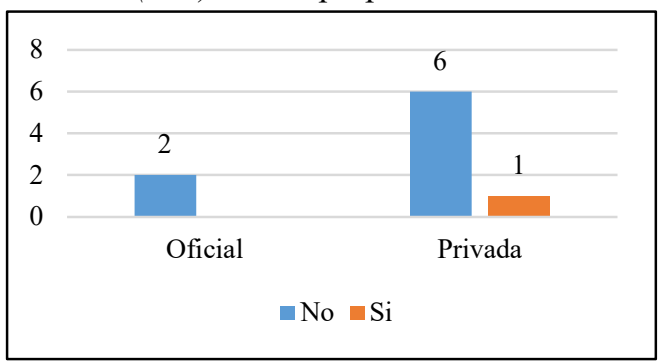

Fuente: SNIES, Colombia. 2017

Los ciclos propedéuticos ofrecen al estudiante la opción de cursar una carrera de forma fragmentada, lo cual permite que éste pueda insertarse en el campo laboral con la posibilidad de continuar a futuro sus estudios en un nivel superior. Para el programa de (SST), se pudo observar que de los nueve (9) programas activos en Colombia, solo existe una (1) oferta para la realización de este programa a través de ciclos propedéuticos y es ofrecida por el sector privado.

\section{i) Programa (SST) Cantidad de Créditos}

Grafica 9: (SST) Cantidad de Créditos. 2018

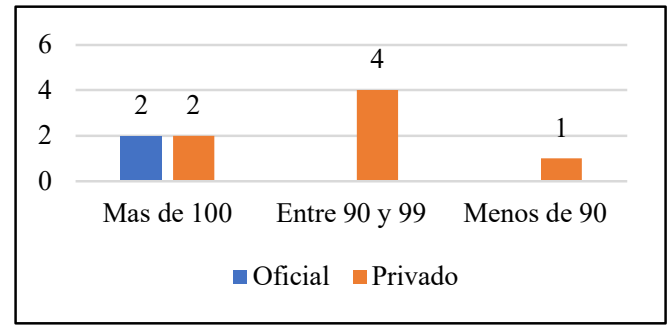

Fuente: SNIES, Colombia. 2018

El crédito es la unidad de medida que representa el tiempo de dedicación del estudiante para el desarrollo de la actividad académica. En concreto, hace referencia a la dedicación con apoyo del docente y al tiempo de trabajo independiente.

Para el programa en cuestión, se pudo observar que de los nueve (9) programas activos, cuatro (4) reportan número de créditos entre 90 y 99; cuatro (4) entre 100 y 110 y solo uno (1) se reportan con menos de 90 créditos.

\section{Conclusiones y Discusión.}

A nivel de distribución nacional, se encontró que de los 26 programas tecnológicos afines reportados en el SNIES, 22 se encuentran activos y 4 inactivos. Del total de los 26 programas relacionados con el área de estudio a nivel nacional, solo 13 corresponden a denominaciones específicas del programa de Tecnología en Salud Ocupacional de los cuales nueve (9) se encuentran activos. Adicionalmente se pudo observar que los 13 restantes corresponden a denominaciones similares y afines, encontrándose activos 13 programas.

En cuanto a la distribución nacional del programa de (TSO) en las cinco (5) regiones del país, se encontró que uno (1) en la región de la Amazonia, cuatro (4) en la Andina, cinco (5) en la Caribe, dos (2) en la Orinoquia y una (1) en la Pacífico. De acuerdo a la información reportada en SNIES, se pudo observar que de los 22 programas de (TSO) activos a nivel nacional, siete (7) corresponden a la modalidad presencial; dos (2) a distancia tradicional. 
Con respecto a la información relacionada con la modalidad empleada por los programas activos (SST) a nivel de regiones, se encontró que solo es ofrecida en la región andina, con tres (3) en modalidad presencial y cuatro (4) virtual. Y en la Región Caribe con dos (2) Presenciales. La sectorización relacionada con un programa académico de cualquier nivel, está relacionada con el tipo de institución educativa que lo ofrece. Así las cosas, se encontró que el programa (SST) a nivel nacional en su gran mayoría es ofertado por instituciones privadas. En concreto se observó que de los nueve (9) programas (TSO) activos, siete (7) son de carácter privado y solamente dos corresponden al sector (2) oficial.

Los ciclos propedéuticos ofrecen al estudiante la opción de cursar una carrera de forma fragmentada, lo cual permite que éste pueda insertarse en el campo laboral con la posibilidad de continuar a futuro sus estudios en un nivel superior. Para el programa de (SST), se pudo observar que, de los nueve (9) programas activos en Colombia, solo existe una (1) oferta para la realización de este programa a través de ciclos propedéuticos y es ofrecida por el sector privado.

\section{Referencias Bibliográficas}

[1] Educación técnica y tecnológica para la competitividad. 2008. [En línea]. Available: www.mineducacion.gov.co/1621/articles 176787 archivo_pdf.pdf. [Último acceso: 07 noviembre 2016]

[2], [3] Panorama de las Carreras Técnicas y Tecnológicas en Colombia. 2005. [En línea]. Available:

http://www.laccei.org/LACCEI2005Cartagena/Pa pers/ED132_MartinezPalmera.pdf. [Último acceso: 10 noviembre 2016]

[4] L. Melo, J. Ramos, P. Hernández «La educación superior en Colombia: situación actual y análisis de eficiencia», Revista Desarrollo y Sociedad. e - ISSN 1900-7760, 2005.

[5], [6], [7], [8] Educación técnica y tecnológica para la competitividad. 2008. [En línea]. Available:

www.mineducacion.gov.co/1621/articles176787_ archivo_pdf.pdf. [Último acceso: 07 noviembre 2016]

\section{Referencias de Apoyo}

Bases de la política para el diseño de programas de educación superior por ciclos y competencias. Ministerio de Educación Nacional. [En línea]. Available:

http://www.mineducacion.gov.co/1621/article131953.html [Último acceso: 07 noviembre 2016]

Alta Consejería Presidencial para la Competitividad y Productividad. [En línea]. Available:

http://www.snc.gov.co/pagina_nueva/consejero/ funciones.html [Último acceso: 07 noviembre 2016]

Bases de la política para el diseño de programas de educación superior por ciclos y competencias. Ministerio de Educación Nacional. [En línea]. Available:

http://www.mineducacion.gov.co/1621/article 131953.html [Último acceso: 07 noviembre 2016]

Comisión Nacional de Competitividad. [En línea]. Available:

http://www.snc.gov.co/pagina_nueva/nacional. html [Último acceso: 07 noviembre 2016]

Comisiones Regionales de Competitividad. [En línea]. Available: http://www.snc.gov.co/pagina_nueva/regionales. $\mathrm{html}$ [Último acceso: 07 noviembre 2016] Convocatoria para apoyar proyectos de transformación de la formación técnica y tecnológica 2007. Ministerio de Educación Nacional. [En línea]. Available: http://www.mineducacion.gov.co/1621/article 127702.html [Último acceso: 07 noviembre 2016]

Departamento Nacional de Planeación. [En línea]. Available: http://www.dnp.gov.co/ [Último acceso: 07 noviembre 2016]

Plan Nacional de Desarrollo. Estado Comunitario: desarrollo para todos. [En línea]. Available: http://www.dnp.gov.co/paginas_detalle.

aspx?idp=699 [Último acceso: 07 noviembre 2016] 